Published in final edited form as:

Oncol Nurs Forum. 2011 November ; 38(6): 672-680. doi:10.1188/11.ONF.672-680.

\title{
Symptom Clusters and Quality of Life in Older Breast Cancer Survivors
}

\author{
Rachel A. Roiland ${ }^{1}$ and Susan M. Heidrich ${ }^{1}$ \\ ${ }^{1}$ School of Nursing, University of Wisconsin-Madison
}

\section{Abstract}

Objective-To identify symptom clusters in older breast cancer survivors and examine whether symptom clusters are related to demographic, health, and quality of life variables.

Design-Symptom bother measures were analyzed using factor analysis to identify possible symptom clusters and the resulting clusters were then correlated with quality of life measures.

Setting - The randomized clinical trail from which the data used for these analyses were drawn took place over the phone between the participant and a trained research nurse.

Sample-Data from 192 older breast cancer survivors (mean age $=70$ ) who had participated in a randomized clinical trial testing a symptom management intervention were used for this study.

Methods-This was a secondary data analysis of the baseline measures of demographics, health history, symptom bother, and physical, mental, and existential dimensions of quality of life. Exploratory and confirmatory factor analyses were conducted as well as MIMIC modeling and partial correlation analyses to assess the relationships amongst clusters and demographic, health history, and quality of life measures.

Main research variables-The main research variables were self-reported symptom bother, demographics such as age and education level, health history and quality of life.

Findings-Seven clinically distinct symptom clusters tapping 36 different symptoms in older breast cancer survivors were found, and these symptom clusters were significantly related to multiple dimensions of quality of life.

Conclusions-Older breast cancer survivors experience multiple, concurrent symptoms that appear to cluster. Identifying symptom clusters helps to elucidate possible inter-symptom relationships, which may lead to the design of more effective symptom management interventions for older breast cancer survivors.

Implications for Nursing-Older breast cancer survivors should be assessed for a wide variety of symptoms if clinicians hope to identify and understand inter-symptom relationships. Such an assessment would enable more comprehensive symptom management.

\section{Keywords}

cancer; older survivors; symptom clusters; quality of life 


\section{Background}

Breast cancer accounts for one in four cancer diagnoses in U.S. women (American Cancer Society (ACS), 2009). Gender and age are the two greatest risk factors for developing the disease, with older women having a higher likelihood of being diagnosed than younger women (ACS, 2009). Between 2003 and 2007, the median age of women diagnosed with breast cancer was 61 and as of January 2007, approximately 2.5 million women in the United States had a history of breast cancer (National Cancer Institute, 2010). That number is only expected to increase as a large cohort of middle aged women move into old age where their risk for breast cancer is increased and, with early detection and improved treatment effectiveness, it is likely these women will survive and live for many years as breast cancer survivors.

As breast cancer survivors age, they can expect to experience a variety of symptoms that may be late effects of cancer, its treatments, or the result of normal aging processes or chronic illnesses (Heidrich, Egan, Hengudomsub, \& Randolph, 2006). In breast cancer survivors, these symptoms have been found to negatively affect well-being and have been associated with increased incidence of depression and anxiety (Kornblith \& Ligibel, 2003; Manning-Walsh, 2005a). Similar relationships have been found in other groups of cancer survivors. Mirabeau-Belle et al. (2009) found fatigue, nausea/vomiting and sleep disturbance in ovarian cancer survivors were correlated with worse emotional states, lower spirituality scores, increased fear of recurrence and lower quality of life. Deimling, Bowman, Sterns, Wagner, and Kahana (2006) observed a strong correlation between increased number of symptoms and worry in a sample of breast, colorectal and prostate cancer survivors, and worry was one of the strongest predictors of anxiety and depression in that same sample.

Effective symptom management may be able to lessen or negate these negative effects and improve breast cancer survivors' quality of life. How to address the multiple symptoms older cancer survivors experience is an important issue. The concept of symptom clusters has emerged as an important topic in recent oncology research, but this research has not adequately addressed the unique symptom experience of older cancer survivors.

Additionally, a focus on symptom clusters may be important in understanding the symptom experience in numerous chronic illnesses or in old age, generally.

Research on symptom clusters has been based on the idea that symptoms are not independent entities but interact with each other and certain symptoms have a tendency to present together or 'cluster'. However, there is little consensus regarding what a symptom cluster is, how to go about identifying and examining symptom clusters analytically, and what the implications are for interventions to improve symptoms. The two most commonly cited definitions are by Dodd, Miaskowski, and Paul (2001) and Kim, McGuire, Tulman, and Barsevick (2005). Dodd et al. (2001) propose, "When three or more concurrent symptoms (e.g. pain, fatigue, sleep insufficiency) are related to each other, they are called a symptom cluster. The symptoms within a cluster are not required to share the same etiology." Kim et al. (2005) require only two concurrent symptoms to be related in order to constitute a symptom cluster and added that these clusters need to be stable and relatively independent of other clusters. The common element to these definitions is that symptoms occur concurrently and are related to each other in some way. What is meant by "related" has not been well articulated, but it may be that clustering symptoms relate to each other via a biological mechanism such as elevated inflammatory processes that result in the presentation of the clustering symptoms (Cleeland et al., 2003). Symptoms may also relate to one another through the degree of burden they inflict on individuals. It may be that symptoms with higher levels of reported burden cluster with each other and interact to 
exacerbate burden levels. Another possibility is that the ways in which symptoms within a cluster relate vary from cluster to cluster necessitating examination of these relationships on a cluster-by-cluster basis.

Studies identifying symptom clusters have utilized different approaches. Some have proposed symptom clusters based on previous empirical research and tested whether the "a priori" symptom clusters were related statistically (Barsevick, Whitmer, Nail, Beck, \& Dudley, 2006; Dodd et al., 2001; Fox \& Lyon, 2006; Fox \& Lyon, 2007). The most common approach has been to assess symptoms using a symptom inventory, such as the M.D. Anderson Symptom Inventory or the Edmonton Symptom Assessment Scale, and then identify symptom clusters using factor or cluster analysis (Chen \& Lin, 2007; Chow, Fan, Hadi, \& Filipsczak, 2007; Gleason et al., 2007; Olson et al., 2008; Sarna \& Brecht, 1997; Wang, Tsai, Chen, Lin, \& Lin, 2008). This last approach may be a better representation of the actual symptom experience because symptoms are assessed systematically.

The impact of symptoms clusters on patient outcomes has also been examined. Miaskowski, Dodd, and Lee (2004) proposed that the effects of symptom clusters on patient outcomes are different than the effects of a single symptom. Patients reporting the presence of multiple symptoms or higher distress from multiple symptoms--such as fatigue, pain, sleep insufficiency and depression-- have poorer functional status compared to patients reporting only one symptom or lower symptom distress scores (Chen \& Tsang, 2006; Dodd et al., 2001; Gaston-Johansson, Fall-Dickson, Bakos, \& Kennedy, 1999; Given, Given, Sikorskii, $\&$ Hadar, 2007). However, it is not known whether this difference is a function of the quantity or quality of the clustering symptoms.

Most research on symptom clusters has been conducted with individuals undergoing treatment for cancer and has focused on treatment-related symptoms. Little symptom cluster research has focused on cancer survivors; particularly older cancer survivors who experience multiple, concurrent symptoms (Heidrich et al., 2006). Further, symptom clusters in cancer survivors, particularly older survivors, may be chronic as opposed to acute treatment-related symptoms. Chronic symptoms have the potential to have a long-term impact on quality of life. Identifying possible symptom clusters and their relationship to patient characteristics and outcomes may lead to a better understanding of older breast cancer survivors' symptom experience which may ultimately lead to better symptom management.

The present study was conducted to address the following gaps in the literature: few symptom cluster studies on cancer survivors, a narrow rather than broad assessment of symptoms, a focus on only acute treatment-related symptoms, and the lack of attention to older adults, who experience more symptoms generally.

The specific aims of this study were to:

1. Identify symptom clusters in older breast cancer survivors

2. Examine whether symptom clusters are related to demographic, health, and quality of life variables

\section{Method} Design

The current study was a secondary analysis of data from a randomized clinical trial of an individualized symptom management intervention for older breast cancer survivors. The parent study tested an eight-week telephone intervention during which an older breast cancer survivor worked with an advanced practice nurse to identify distressing symptoms, identify 
and discuss beliefs regarding those symptoms, and develop personalized goals and plans for symptom management. Data regarding demographics, health history, quality of life, and number of and distress from symptoms were collected at baseline and were used for this study. The parent study was approved by the Health Sciences Institutional Review Board.

\section{Sample}

Eligibility criteria for participation in the parent study were: women aged 65 or older, at least one year since breast cancer diagnosis, at least 3 months post treatment (except adjuvant hormonal therapies), and no recurrent or metastatic disease or other cancer diagnosis. A total of 192 participants completed the baseline measurements used for this analysis

\section{Instruments}

Demographic and health history-Participants were asked about their age, education, marital status, living arrangements, ethnicity, and income. Information regarding date of breast cancer diagnosis, past treatments for breast cancer and dates of treatment was also collected. Number of chronic illnesses was measured with the Wisconsin Longitudinal Study of Families Form F. Respondents identified whether they had been told by a healthcare provider that they had any of 20 health problems, and the total number of health problems was computed.

Symptom Distress-Symptoms were assessed with the Symptom Bother Scale-Revised (SB-R) (Heidrich et al, 2006). The 37-item scale includes symptoms common to old age, chronic conditions common in old age, and late effects of breast cancer and its treatment. Participants were asked to what degree they are bothered by each symptom. The instrument uses a 0 (don't have the symptom), 1(have, but am not bothered) to 5 (extremely bothered) scale. The scale has been found to be reliable and valid in studies with older adults and adults with cancer with reported reliability coefficients (Cronbach's alpha) ranging from 0.78 to 0.89 (Heidrich, 1993, 1994, 1998, 2006). In the current study, total number of symptoms (0-37) and mean symptom distress were calculated, and Cronbach's alpha was 0.91 .

Quality of Life-Four instruments were used to measure the physical, emotional and existential dimensions of quality of life.

The Medical Outcomes Study Short Form-36 (SF-36) is a 36-item scale containing two subscales measuring physical and mental quality of life (Ware \& Sherbourne, 1992). These two subscales capture the extent to which physical or mental health problems interfere with different domains of life. Scores are standardized so that higher scores indicate less interference from physical or mental health problems and therefore better physical or mental quality of life (QOL). The instrument has been found to be reliable and valid in previous studies of older patients and patients with breast cancer with reported reliability coefficients ranging from 0.62 to 0.90 (Schlenk et al., 1998; Ware \& Sherbourne, 1992). In the current study, Cronbach's alphas were 0.93 and 0.91 for physical and mental QOL subscales respectively.

Existential quality of life was measured with two scales: the 14-item Purpose in Life Scale (PIL) scale and the 14-item Positive Relations with Others Scale (PR) scale, two of Ryff's Psychological Well Being scales (1989). The PIL Scale measures the degree to which one's life has meaning, direction, and goals. Purpose in life has been shown to be important in the quality of life and psychological adjustment of breast cancer survivors (Jim \& Anderson, 2007; Manning- Walsh, 2005b; Stanton, Danoff-Burg \& Huggins, 2002; Stanton, Simonelli, 
Fowler, Maxwell, \& Andersen, 2008). The Positive Relations Scale taps important dimensions of social support, particularly emotional support. Both scales have been used in cross-sectional, longitudinal, and cross-cultural studies and are related to other indices of well-being (i.e., affect balance, life satisfaction, self-esteem, morale, depression, and internal locus of control). For each scale, participants respond to items on a 1 (strongly disagree) to 6 (strongly agree) scale. Higher scores indicate higher levels of purpose in life or more positive relationships with others. In the current study, Cronbach's alphas were 0.88 for both scales.

Depressive symptoms were measured using the 10-item version of the Center for Epidemiologic Studies-Depression Scale (CES-D) (Radloff, 1977). This scale has been tested and validated in a number of studies of older adults (Irwin, Artin, \& Oxman, 1999; Kohout, Berkman, Evans, \& Cornoni-Huntley, 1993). The respondents answer yes (1) or no (0) to whether they experienced a given symptom of depression much of the time during the past week. Higher scores indicate more depressive symptoms. The short version, using a cut-off score of 4 for clinical depression, has a sensitivity of $97 \%$, specificity of $84 \%$, and positive predictive value of $85 \%$ in older adults. In this study Cronbach's alpha was 0.65 . The lower reliability may be due to the dichotomous response scale.

\section{Data Analysis}

To identify symptom clusters, symptom bother scores for each of the 37 symptoms from the baseline SB-R were analyzed using exploratory and confirmatory factor analysis. Exploratory factor analysis (EFA) was conducted first with maximum likelihood as the estimator and quartimin (oblique) rotation. Symptoms were retained if the symptom had a factor loading greater than or equal to 0.20 on any of the factors. Generally, the cut-off criteria for factor loadings is between 0.3 and 0.4 but, given the exploratory nature of this study, 0.20 was used in order to include the largest number of symptoms in the analysis so as to better represent the symptom experience of the study participants. The factor structure was then tested using a confirmatory factor analysis (CFA). In the CFA, each symptom was assigned to the factor on which it had the highest loading in the EFA. Three fit indices were used to determine the adequacy of the model's fit with the data. For the Chi-square to degrees of freedom ratio $\left(\mathrm{X}^{2} / \mathrm{df}\right)$, a ratio less than or equal to three indicates a good fit for the data (Bollen, 1989). For the Comparative Fit Index (CFI), a CFI of 0.95 or greater indicates a good fit and for the Tucker Lewis Index (TLI), a TLI of 0.95 or greater indicates good fit (Hu \& Bentler, 1999). Both the exploratory and confirmatory factory analyses were completed using the statistical package Mplus Version 5 (Muthén \& Muthén, 1998).

To examine relationships among clusters and demographic and health history variables, multiple indicator multiple cause (MIMIC) structural equation models were used. A MIMIC model allows for the regression of latent variables, such as factors (in this case, the symptom clusters), on to grouping variables that can be either continuous or categorical (Woods, Oltmanns, \& Turkheimer, 2009). The MIMIC model analyses were completed using Mplus Version 5 (Muthén \& Muthén, 1998).

Relationships among factors and quality of life variables were examined by first calculating symptom cluster scale scores by averaging the individual symptom SB-R ratings for the clusters that were identified in the CFA. The scale scores were then correlated with quality of life variables to identify possible significant relationships. This analysis was done using the statistical program SPSS version 16.0 (SPSS, Inc., Chicago, IL, 2007). 


\section{Results}

\section{Demographic and Clinical Characteristics}

As shown in Table 1, approximately $94 \%$ of participants were Caucasian, $58 \%$ were married, and the mean age was 70 years. The average time since diagnosis was less than five years, and mean time since last treatment was 34.8 months. Past cancer treatments included mastectomy (39\%), lumpectomy (67\%), radiation (70\%), chemotherapy $(38 \%)$ and hormonal therapies $(67 \%)$. Participants reported an average 6 chronic conditions with arthritis/rheumatism (74\%), high cholesterol (59\%), and high blood pressure (57\%) being the most prevalent.

\section{Symptom Frequency and Bother}

Participants reported an average of 17 symptoms $(\mathrm{SD}=7.6)$. The frequency and mean bother scores for each of the 37 symptoms are shown in Table 2 . The 5 most frequently reported symptoms were: stiffness (88\%), pain (85\%), joint pain (82\%), aching (80\%) and fatigue $(78 \%)$. Mean symptom bother scores ranged from 2.30 for pain to 0.20 for vaginal discharge. The five most bothersome symptoms reported by participants were pain, joint pain, stiffness, aching, and fatigue.

\section{Exploratory Factor Analysis}

Results from the exploratory factor analysis indicated a seven factor solution was the most acceptable. The fit indices for this model were: $\mathrm{X}^{2} / \mathrm{df}=1.62, \mathrm{p}=0.001, \mathrm{CFI}=0.90$, and TLI $=0.85$. The CFI and TLI were below the "good fit" values, but the solution warranted further examination because the factors were clinically interpretable. Only one symptom (vaginal discharge) was dropped because of factor loadings $<0.20$.

\section{Confirmatory Factory Analysis}

The seven factor solution from the EFA was tested with confirmatory factor analysis using a maximum likelihood estimation and quartimin (oblique) rotation. Results are reported in Table 3. The fit indices were $\mathrm{X}^{2} / \mathrm{df}=1.82, \mathrm{p}=0.001$, CFI $=0.821$, and TLI $=0.803$. Overall, these indices indicate an adequate fit of the model to the data. The seven factors were labeled: musculoskeletal symptoms, neurocognitive symptoms, dryness symptoms, urinary symptoms, circulatory symptoms, sleep symptoms, and hormonal symptoms.

In this model, all standardized factor loadings were significant indicating that the items were appropriately assigned to the factors and were sufficient indicators of the latent variable underlying each cluster. In addition, the $\mathrm{R}^{2}$ was greater than .20 for 32 of the 36 symptoms, indicating the item is a reliable indicator of the factor. Four symptoms had an $\mathrm{R}^{2}$ less than 0.20 (vaginal dryness, hot flashes, decreased sex drive, and shortness of breath), but were retained because at least fifty percent of the sample reported having these symptoms.

Symptom cluster scale scores were computed by averaging the SB-R bother mean scores for each item loading on the cluster. The two most bothersome clusters were the musculoskeletal and sleep clusters with mean bother scores of 1.98 and 1.40 respectively. Descriptive statistics and reliability estimates (alpha) for each scale are reported in Table 3.

\section{Relationship of symptom clusters to demographic, health history and quality of life}

MIMIC models were used to test whether any demographic or clinical variables (i.e. past cancer treatments) were significantly related to any of the symptom cluster scale scores. For demographic variables, the only significant relationship was between years of education and the circulatory cluster $(\gamma=0.002, p=0.006)$. For health history, number of chronic illnesses 
was significantly related to all seven symptom clusters: musculoskeletal $(\gamma=0.23 ; \mathrm{p}=$ $0.0001)$, neurocognitive $(\gamma=0.17 ; \mathrm{p}=0.0001)$, dryness $(\gamma=0.14 ; \mathrm{p}=0.0001)$, urinary $(\gamma=$ $0.18 ; \mathrm{p}=0.0001)$, circulatory $(\gamma=0.215 ; \mathrm{p}=0.0001)$, sleep $(\gamma=0.15 ; \mathrm{p}=0.0001)$ and hormonal $(\gamma=0.11 ; p=0.0001)$. Having had a mastectomy was significantly related to the hormonal cluster $(\gamma=0.740 ; p=0.021$ ), and past lumpectomy (but not radiation) was significantly related to the neurocognitive cluster $(\gamma=0.528 ; \mathrm{p}=0.042)$.

\section{Relationship between symptom clusters and quality of life}

Partial correlations were computed between the symptom cluster scale scores and the quality of life measurements, controlling for number of medications, age, years of educations, family income, and number of chronic conditions. As shown in Table 4, higher levels of depression and poorer mental QOL were significantly related to higher symptom bother from all seven symptom clusters. Worse physical quality of life was associated with higher symptom bother from all but the hormonal symptoms. Less purpose in life was associated with higher symptom bother from all but the sleep symptoms, and less positive relations were associated with higher symptom bother from all but the musculoskeletal and sleep symptoms.

\section{Discussion}

Seven clinically distinct symptom clusters tapping 36 different symptoms in older breast cancer survivors were found, and these symptom clusters were significantly related to multiple dimensions of quality of life. The finding that there are clinically distinct symptom clusters may indicate a biological mechanism is linking the symptoms within a cluster together and distinguishing the clusters from each other. Uncovering biological mechanisms is one of the goals of symptom cluster research because such mechanisms suggest that treatment for one symptom may be efficacious in treating all symptoms in the cluster (Miaskowski \& Aouizerat, 2007). The "Sickness Behavior Model" has been proposed to explain a biological mechanism for the symptoms observed in individuals with cancer or who are undergoing treatment for cancer (Cleeland et al., 2003). The model suggests that the presence of cancerous tumors leads to increased production of pro-inflammatory cytokines such as interlukin-1 and interlukin-6. In animal models, increased production of these cytokines has been associated with fever, pain, somnolence, and decreased activity. Increased levels of these pro-inflammatory cytokines have also been found to be related to changes in measurements of depression and quality of life in cancer patients who had not yet started treatment (Rich, 2007). It is not clear whether the Sickness Behavior Model can explain the numerous and often chronic symptoms experienced by older cancer survivors.

A major issue is whether the symptom clusters identified in these older breast cancer survivors are due to the individual's past cancer and cancer treatment or to normal aging and age-related chronic health problems. In this study, significant relationships were noted between mastectomy and the hormonal cluster and lumpectomy and the neurocognitive cluster, while number of chronic conditions was significantly related to all seven symptom clusters. The explanations for these relationships are not clear, but it would be informative to compare symptom clusters in cancer and non-cancer groups over time to see if clusters change from treatment through survivorship or differ by diagnosis. Such an examination might provide a clearer explanation for relationships between past treatment and current symptoms in older cancer survivors. Future studies will need to further examine the possible mechanisms underlying symptom clusters in order to better understand how and when symptoms interact.

Age was significantly related to two clusters (neurological and dryness), even with the restricted age range in this sample. This underscores the importance of taking age into 
account in research on symptoms, since so many symptoms are age-related. Ignoring the influence of age can lead to erroneous causal inferences; that is, that a symptom cluster is related to the underlying diagnosis (in this case, breast cancer) rather than the aging process or other unknown age-related health problems. On the other hand, number of chronic conditions was significantly related to all of the clusters, underscoring the importance of taking co-morbidities into account in studies of older adults in order to avoid erroneous conclusions about the effect of age, per se.

The results of this study provide further evidence for the relationship between symptom experience and quality of life. In this study, the musculoskeletal cluster and physical quality of life were highly correlated. This relationship makes intuitive sense given the symptoms in this cluster (aching, weakness, joint pain, stiffness, pain, and fatigue) would make it difficult to be physically active or carry out physical activities of daily living. Another particularly strong relationship was observed between the hormonal cluster (mood swings, depression, anxiety, nightmares, hot flashes, headache, weight gain and/or loss, vaginal dryness) and mental quality of life. This finding is consistent with other studies finding hot flashes to be more distressing and severe in breast cancer survivors compared to women with no cancer history (Carpenter, Johnson, Wagner \& Andrykowski, 2002). Hot flashes have also been related to greater sleep disturbance and interference with activities of daily living, as well as poorer quality of life (Carpenter et al., 2002; Gupta et al., 2006; Rand et al., 2010). A study by Schultz, Klein, Beck, Stava, and Sellin (2005) found significant relationships between menopausal symptoms such as hot flashes and painful intercourse (a possible indication of vaginal dryness) and unhappiness, fatigue and difficulty concentrating. However, for the current study it is important to note there may be some measurement overlap given that the symptom of depression loaded on the hormonal cluster and the mental quality of life subscale taps depressed mood.

Other dimensions of quality of life were also found to have significant relationships with several of the symptom clusters. Purpose in life was significantly related to all of the clusters except for the sleep cluster. Previously, purpose in life in men and women over 85 has been shown to be negatively affected by musculoskeletal symptoms, and older adults reporting low levels of purpose in life experienced earlier death compared to individuals with high levels of purpose in life (Boyle, Barnes, Aron, \& Bennett, 2009; Hedberg, Gustafson, \& Brulin, 2010). Positive relationships were also significantly correlated with the majority of the symptom clusters; a finding consistent with Manning-Walsh (2005b) who found that positive relations were negatively related to symptom distress in breast cancer survivors. Purpose in life and positive relationships with others have been found to be important components of quality of life in old age in the general population (Borg, Hallber \& Blomqvist, 2006; Bowling et al., 2002; Low \& Molzahn, 2007). The significant relationships among symptom clusters and quality of life measures observed in the current study illustrate the far reaching effects symptoms can have on multiple dimensions of breast cancer survivors' quality of life and emphasizes the importance of adequate symptom management in this population.

\section{Limitations}

The first limitation to this study is that the cross-sectional design precludes the ability to determine causal relationships. For instance, it may be that the symptom clusters influenced quality of life or it may be that quality of life influenced either the experience or reporting of different symptoms, or the relationships may be reciprocal. On the other hand, we do know that previous cancer treatments, such as mastectomy and lumpectomy, were related to some current symptom clusters. Because this was a cross-sectional study, we also could not address the temporal nature of how symptoms are experienced; that is, symptoms often wax 
and wane over time. Future studies should incorporate a longitudinal design to better understand those issues.

A second limitation is that the sample for this study included only women who had been diagnosed and treated for breast cancer. The majority of participants was Caucasian and somewhat highly educated. The results of this study may not be generalizable to men, different racial/ethnic groups, or to persons with other types of cancers. On the other hand, the women in this study were diverse in terms of socioeconomic status and rural versus urban communities. In addition, they were similar to older women generally in terms of their overall health status.

A final limitation is the use of self-report data that can be associated with mono-method bias and attenuated correlations. Future studies of symptom clusters would benefit from the addition of biophysical measures, particularly if underlying biological mechanisms of symptom clusters are to be determined.

\section{Conclusions}

Seven symptom clusters were identified and were found to be significantly related to multiple dimensions of quality of life in older breast cancer survivors. Older breast cancer survivors experience multiple symptoms concurrently and should be screened for a wide variety of symptoms if clinicians hope to provide comprehensive symptom management. Identifying symptom clusters may help to elucidate inter-symptom relationships, which may lead to the design of more effective interventions that can target symptoms known to be related to one another and may result in greater improvements in patient outcomes such as quality of life.

\section{Implications for Practice}

Thorough symptom assessment should be routine for breast cancer survivors if clinicians hope to provide comprehensive symptom management. Given the number of symptoms identified by participants in this study (an average of 17), these symptom assessments should be comprehensive, including symptoms common to old age as well as symptoms common in breast cancer survivors since these symptoms may interact and thereby cause greater burden to the individual. The assessments should be incorporated into the care provided by nurses and physicians in both oncology and primary care to ensure that changes in symptom occurrence, burden, and severity are identified, and interventions are developed. Furthermore, communication between primary care providers and oncology specialists in caring for older cancer survivors is essential to being able to assess the etiology of symptoms that can be ambiguous in older cancer survivors. A yearly assessment of symptoms and educating patients to report any new, worrisome symptom would be important in determining whether a change in symptoms is due to aging, chronic health problems, or a new or recurrent cancer. Developing more effective and efficient symptom management may lead to better quality of life for breast cancer survivors.

\section{Acknowledgments}

The project described was supported by Award Number R01AG022914 from the National Institute on Aging and the Office of Cancer Survivorship, National Cancer Institute

\section{References}

American Cancer Society. Breast Cancer Facts and Figures, 2009-2010. Atlanta: American Cancer Society; 2009. 
Barsevick AM, Whitmer K, Nail LM, Beck SL, Dudley W. Symptom cluster research: Conceptual, design, measurement, and analysis issues. Journal of Pain and Symptom Management. 2006; 31(1): 85-95.10.1016/j.jpainsymman.2005.05.015 [PubMed: 16442485]

Bollen, KA. Structural equation models with latent variable. New York, NY: John Wiley \& Sons; 1989.

Borg C, Hallberg IR, Blomqvist K. Life satisfaction among older people (65+) with reduced self-care capacity: the relationship to social, health and financial aspects. Journal of Clinical Nursing. 2006; 15(5):607-618. [PubMed: 16629970]

Bowling A, Banister D, Sutton S, Evans O, Windsor J. A multidimensional model of the quality of life in older age. Ageing \& Mental Health. 2002; 6(4):335-371.10.1080/1360786021000006983

Boyle PA, Barnes LL, Buchman AS, Bennett DA. Purpose in life is associated with mortality among community-dwelling older persons. Psychosomatic Medicine. 2009; 71(5):574-579.10.1097/PSY. 0b013e3181a5a7c0 [PubMed: 19414613]

Carpenter JS, Johnson DH, Wagner LJ, Andrykowski MA. Hot flashes and related outcomes in breast cancer survivors and matched comparison women. Oncology Nursing Forum. 2002; 29(3):E16E25.10.1188/02.ONF.E16-E25 [PubMed: 11979290]

Chen M, Tseng H. Symptom clusters in cancer patients. Supportive Care Cancer. 2006; 14(8):825830.10.1007/500520-006-0019-8

Chen M, Lin C. Cancer symptom clusters: A validation study. Journal of Pain and Symptom Management. 2007; 34(6):590-599.10.1016/j.jpainsymman.2007.01.008 [PubMed: 17629670]

Chow E, Fan G, Hadi S, Filipczak L. Symptom clusters in cancer patients with bone metastases. Supportive Care in Cancer. 2007; 15(9):1035-1043.10.1007/s00520007-0241-z [PubMed: 17394024]

Cleeland CS, Bennett GJ, Dantzer R, Dougherty PM, Dunn AJ, Meyers CA, Miller AH, Payne R, Reuben JM, Wang XS, Lee B. Are the symptoms of cancer and cancer treatment due to a shared biologic mechanism? A cytokine - immunologic model of cancer symptoms. Cancer. 2003; 97(11):2919-2925.10.1002/cncr.11382 [PubMed: 12767108]

Dodd MJ, Miaskowski C, Paul SM. Symptom clusters and their effect on the functional status of patients with cancer. Oncology Nursing Forum. 2001; 28(3):465-470. [PubMed: 11338755]

Deimling GT, Sterns S, Bowman KF, Kahana B. The health of older-adult, long-term cancer survivors. Cancer Nursing. 2005; 28(6):415-424. [PubMed: 16330962]

Deimling GT, Bowman KF, Sterns S, Wagner LJ, Kahana B. Cancer-related health worries and psychological distress among older adult, long-term cancer survivors. Psycho-Oncology. 2006; 15:306-320.10.1002/pon.955 [PubMed: 16041841]

Deimling GT, Bowman KF, Wagner LJ. The effects of cancer-related pain and fatigue on functioning of older adult, long-term cancer survivors. Cancer Nursing. 2007; 30(6):421433.10.1097/01.NCC.0000300168.88089.2b [PubMed: 18025913]

Fox SW, Lyon DE. Symptom clusters and quality of life in survivors of lung cancer. Oncology Nursing Forum. 2006; 33(5):931-936.10.1188/06.ONF.931-936 [PubMed: 16955121]

Fox SW, Lyon D. Symptom clusters and quality of life in survivors of ovarian cancer. Cancer Nursing. 2007; 30(5):354-361. [PubMed: 17876181]

Gaston-Johansson F, Fall-Dickson JM, Bakos AB, Kennedy MJ. Fatigue, pain, and depression in preautotransplant breast cancer patients. Cancer Practice. 1999; 7(5):240-247.10.1046/j. 1523-5394.1999.75008.x [PubMed: 10687593]

Given BA, Given CW, Sikorskii A, Hadar N. Symptom clusters and physical function for patients receiving chemotherapy. Seminars in Oncology Nursing. 2007; 23(2):121-126.10.1016/j.soncn. 2007.01.005 [PubMed: 17512439]

Gleason JF, Case D, Rapp SR, Ip E, Naughton M, Butler JM, McMullen K, Stieber V, Saconn P, Shaw EG. Symptom clusters in patients with newly diagnosed brain tumors. The Journal of Supportive Oncology. 2007; 5(9):427-436. [PubMed: 18019850]

Gupta P, Sturdee DW, Palin SL, Majumder K, Fear R, Marshall T, Paterson I. Menopausal symptoms in women treated for breast cancer: The prevalence and severity of symptoms and their perceived effects on quality of life. Climateric. 2006; 9(1):49-58.10.1080/13697130500487224 
Hedberg P, Gustafson Y, Brulin C. Purpose in life among men and women aged 85 years and older. International Journal of Aging and Human Development. 2010; 70(3):213-229.10.2190/AG.70.3.c [PubMed: 20503806]

Heidrich SM. The relationship between physical health and psychological well-being in elderly women: A developmental perspective. Research in Nursing and Health. 1993; 16(2):123130.10.1002/nur.4770160207 [PubMed: 8502764]

Heidrich SM. The self, health, and depression in elderly women. Western Journal of Nursing Research. 1994; 16(5):544-555.10.1177/019394599401600507 [PubMed: 7992492]

Heidrich SM. Older women's lives through time. Advances in Nursing Science. 1998; 20(3):65-75. [PubMed: 9504209]

Heidrich SM, Egan JJ, Hengudomsub P, Randolph SM. Symptoms, symptom beliefs, and quality of life of older breast cancer survivors: A comparative study. Oncology Nursing Forum. 2006; 33(2): 315-322.10.1188/06.ONF.315-322 [PubMed: 16518447]

$\mathrm{Hu}$ L, Bentler PM. Cutoff criteria of fit indexes in covariance structure analysis: Conventional criteria versus new alternatives. Structural Equation Modeling. 1999; 6(1):155.10.1080/10705519909540118

Irwin M, Artin LH, Oxman MN. Screening for depression in the older adult: Criterion validity for 10item Center for Epidemiological Studies Depression Scale (CES - D). Archives of Internal Medicine. 1999; 159(15):1701-1704. [PubMed: 10448771]

Jim HS, Andersen BL. Meaning in life mediates the relationship between social and physical functioning and distress in cancer survivors. British Journal of Health Psychology. 2007; 12(3): 363-381.10.1348/135910706X128278 [PubMed: 17640452]

Kim HJ, McGuire DB, Tulman L, Barsevick. Symptom clusters: Concept analysis and clinical implications for cancer nursing. Cancer Nursing. 2005; 28(4):270-282. [PubMed: 16046888]

Kohout FJ, Berkman LF, Evans DA, Cornoni-Huntley J. Two shorter forms of the CES - D (Center for Epidemiological Studies Depression) depression symptoms index. Journal of Aging and Health. 1993; 5(2):179-193.10.1177/089826439300500202 [PubMed: 10125443]

Kornblith AB, Ligibel J. Psychosocial and sexual functioning of survivors of breast cancer. Seminars in Oncology. 2003; 3(6):799-813.10.1053/j.seminoncol.2003.08.025 [PubMed: 14663780]

Low G, Molzahn AE. Predictors of quality of life in old age: A cross - validation study. Research in Nursing \& Health. 2007; 30(2):141-150.10.1002/nur.20178 [PubMed: 17380515]

Manning-Walsh L. Psychospiritual well-being and symptom distress in women with breast cancer. Oncology Nursing Forum. 2005a; 32(3):E56-E62.10.1188/05.ONF.E56-E62

Manning-Walsh J. Spiritual struggle: effect on quality of life and life satisfaction in women with breast cancer. Journal of Holistic Nursing. 2005b; 23(2):120-140.10.1177/0898010104272019 [PubMed: 15883461]

Miaskowski C, Dodd M, Lee K. Symptom clusters: The new frontier in symptom management research. Journal of the National Cancer Institute Monographs. 2004; (32):17-21.10.1093/ jncimonographs/lgh023 [PubMed: 15263036]

Miaskowski C, Aouizerat BE. Is there a biological basis for the clustering of symptoms? Seminars in Oncology Nursing. 2007; 23(2):99-105.10.1016/j.soncn.2007.01.008 [PubMed: 17512436]

Mirabeau-Beale KL, Kornblith AB, Penson RT, Lee H, Goodman A, Campos SM, Matulonis UA. Comparison of the quality of life and advanced stage ovarian cancer survivors. Gynecologic Oncology. 2009; 114(2):353-359.10.1016/j.ygyno.2009.05.009 [PubMed: 19481244]

Muthén, LK.; Muthén, BO. Mplus user's guide. Vol. 2007. Los Angeles, CA: Muthén \& Muthén; 1998.

National Cancer Insitute. SEER stat fact sheet: Breast. 2010. Retrieved January $29^{\text {th }}$, 2010 from: http:// seer.cancer.gov/statfacts/html/breast.html

Olson K, Hayduk L, Cree M, Cui Y, Quan H, Hanson J, Lawlor P, Strasser F. The changing causal foundations of cancer-related symptom clustering during the final month of palliative care: A longitudinal study. BioMed Central Medical Research Methodology. 2008; 8:3647.10.1186/1471-2288-8-36 [PubMed: 18533033]

Radloff L. The CES-D Scale: A self report depression scale for research in the general population. Applied Psychological Measurement. 1977; 1(3):385-401.10.1177/014662167700100306 
Rand KL, Otte JL, Flockhart D, Hayes D, Storniolo AM, Stearns V, Carpenter JS. Modeling hot flashes and quality of life in breast cancer survivors. Climacteric. 2010 Advance online publication. 10.3109/13697131003717070

Rich TA. Symptom clusters in cancer patients and their relations to EGFR ligand modulation of the circadian axis. The Journal of Supportive Oncology. 2007; 5(4):167-174. [PubMed: 17500504]

Ryff CD. Happiness is everything, or is it? Explorations on the meaning of psychological well-being. Journal of Personality and Social psychology. 1989; 57(6):10691081.10.1037/0022-3514.57.6.1069

Sarna L, Brecht M. Dimensions of symptom distress in women with advanced lung cancer: A factor analysis. Heart \& Lung. 1997; 26(1):23-30.10.1016/S0147-9563(97)90006-6 [PubMed: 9013218]

Schlenk EA, Erlen JA, Dunbar-Jacob J, McDowell J, Engberg S, Sereika Bernier MJ. Health-related quality of life in chronic disorders: A comparison across studies using the MOS SF-36. Quality of Life Research. 1998; 7(1):57-65.10.1023/A:1008836922089 [PubMed: 9481151]

Schultz PN, Klein MJ, Beck ML, Stava C, Sellin RV. Breast cancer: Relationship between menopausal symptoms, physiologic health effect of cancer treatment and physical contestants on quality of life in long-term survivors. Journal of Clinical Nursing. 2005; 14(2):204-211.10.1111/j. 1365-2702.2004.01030.x [PubMed: 15669929]

Simonelli LE, Fowler J, Maxwell GL, Andersen BL. Physical sequelae and depressive symptoms in gynecologic cancer survivors: meaning in life as a mediator. Annals of Behavioral Med. 2008; 35(3):275-284.10.1007/s12160-008-9029-8.

Stanton AL, Danoff-Burg S, Huggins ME. The first year after breast cancer diagnosis: Hope and coping strategies as predictors of adjustment. Psycho-Oncology. 2002; 11(2):93-102.10.1002/pon. 574 [PubMed: 11921325]

Wang S, Tsai C, Chen B, Lin C, Lin C. Symptom clusters and relationships to symptom interference with daily life in Taiwanese lung cancer patients. Journal of Pain and Symptom Management. 2008; 35(3):258-266.10.1016/j.jpainsymman.2007.03.017 [PubMed: 18201865]

Ware JE Jr, Sherbourne CD. The MOS 36-item short-form health survey (SF-36). I. Conceptual framework and item selection. Medical Care. 1992; 30(6):473-483. [PubMed: 1593914]

Woods CM, Otlmanns TF, Turkheimer E. Illustration of MIMIC-Model DIF testing with the Schedule for Nonadaptive and Adaptive Personality. Journal of Psychopathology and Behavioral Assessment. 2009; 31(4):320-330.10.1007/s10862-008-9118-9 [PubMed: 20442793] 
Table 1

Summary of Demographic and Health History Characteristics ( $\mathrm{n}=192)$

\begin{tabular}{|c|c|c|c|}
\hline Variables & M (SD) & Range & Frequency $(\%)$ \\
\hline Age & $70(5.30)$ & $65-97$ & \\
\hline Education (years) & $14(2.61)$ & $10-22$ & \\
\hline \multicolumn{4}{|l|}{ Race/ Ethnicity } \\
\hline Non-Hispanic White & & & $180(94)$ \\
\hline American Indian/ Alaska Native & & & $1(0.5)$ \\
\hline Asian/ Pacific Islander & & & $1(0.5)$ \\
\hline African American & & & $8(4)$ \\
\hline Hispanic & & & $2(1)$ \\
\hline Married & & & $111(58)$ \\
\hline \multicolumn{4}{|l|}{ Total family annual income (\$) } \\
\hline$<25,000$ & & & $47(24)$ \\
\hline 25,000 to 54,999 & & & $75(39)$ \\
\hline 55,000 to 84,999 & & & $30(15)$ \\
\hline 85,000 and over & & & $18(9)$ \\
\hline Missing & & & $20(10)$ \\
\hline \multicolumn{4}{|l|}{ Living arrangements } \\
\hline Living alone & & & $64(33)$ \\
\hline Living with others & & & $116(60)$ \\
\hline Number of chronic illnesses & $6(5.93)$ & $2-14$ & \\
\hline Number of symptoms & $17(7.60)$ & $3-37$ & \\
\hline Months since diagnosis of breast cancer & $39(40.92)$ & $12-266$ & \\
\hline \multicolumn{4}{|l|}{ Types of cancer treatment ${ }^{a}$} \\
\hline Mastectomy & & & $75(39)$ \\
\hline Lumpectomy & & & $129(67)$ \\
\hline Radiation & & & $135(70)$ \\
\hline Chemotherapy & & & $72(37)$ \\
\hline Hormonal therapy & & & $129(67)$ \\
\hline
\end{tabular}

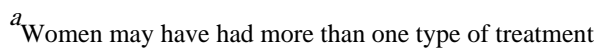


Table 2

Symptom Occurrence and Bother

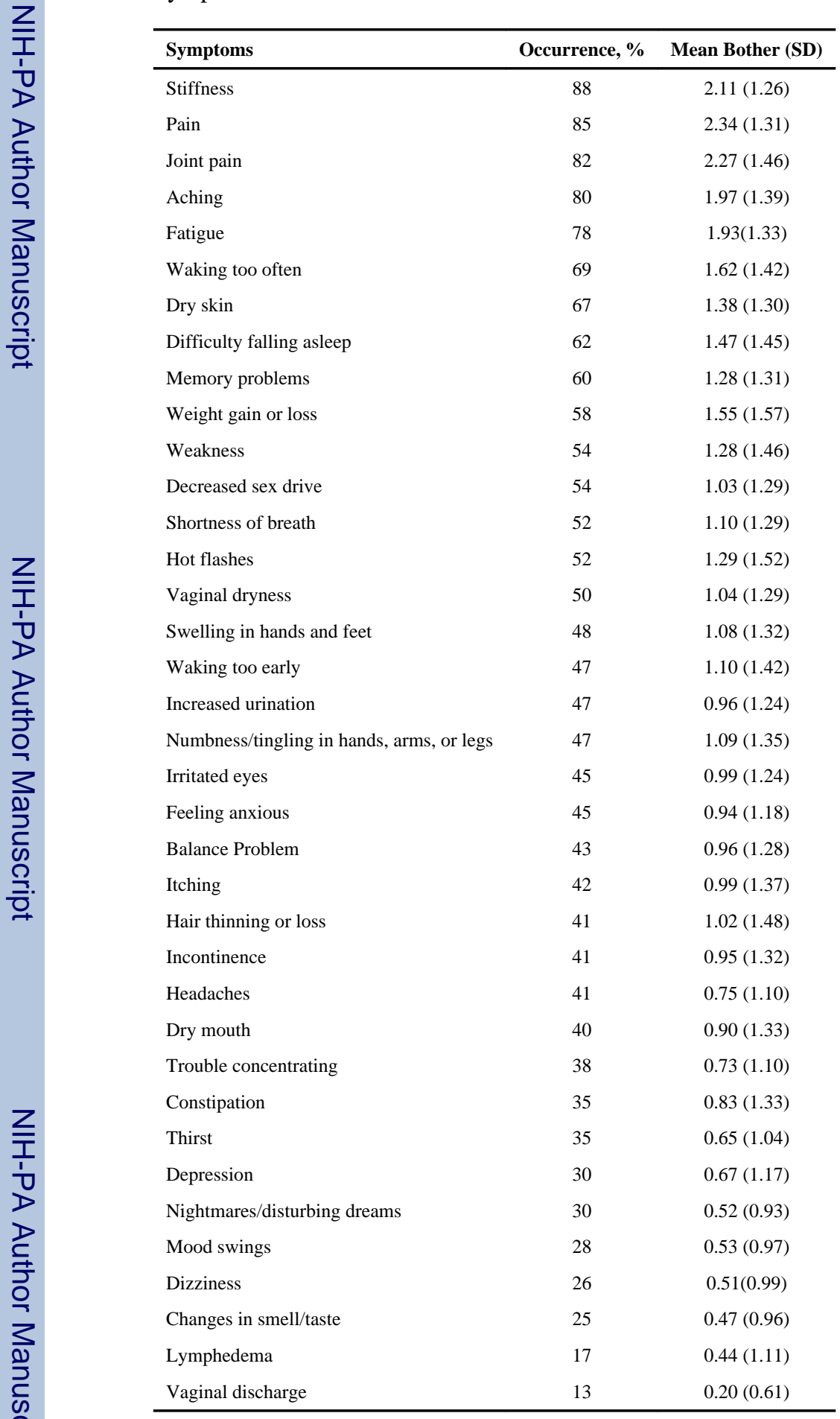


Table 3

Confirmatory Factor Analysis Loadings, Factor Alphas and Mean Symptom Bother Scores

\begin{tabular}{|c|c|c|c|c|}
\hline Factors/Items & Standardized Loading $a$ & Standard Error & Factor Alpha & Mean bother (SD) \\
\hline Musculoskeletal & & & 0.89 & $1.98(1.10)$ \\
\hline Aching & 1.18 & 0.08 & & \\
\hline Stiffness & 0.99 & 0.08 & & \\
\hline Pain & 0.97 & 0.08 & & \\
\hline Joint pain & 1.05 & 0.09 & & \\
\hline Weakness & 1.13 & 0.09 & & \\
\hline Fatigue & 0.95 & 0.09 & & \\
\hline Neurocognitive & & & 0.81 & $0.87(0.94)$ \\
\hline Balance problem & 0.88 & 0.09 & & \\
\hline Dizziness & 0.58 & 0.07 & & \\
\hline Memory problems & 1.03 & 0.08 & & \\
\hline Trouble concentrating & 0.90 & 0.07 & & \\
\hline Dryness & & & 0.69 & $1.09(0.85)$ \\
\hline Dry skin & 0.77 & 0.10 & & \\
\hline Itching & 0.87 & 0.10 & & \\
\hline Dry Mouth & 0.82 & 0.10 & & \\
\hline Thirst & 0.59 & 0.08 & & \\
\hline Shortness of breath & 0.55 & 0.10 & & \\
\hline Urinary & & & 0.98 & $0.98(0.84)$ \\
\hline Incontinence & 1.06 & 0.10 & & \\
\hline Increased urination & 0.95 & 0.09 & & \\
\hline Decreased sex drive & 0.23 & 0.10 & & \\
\hline Irritated eyes & 0.41 & 0.01 & & \\
\hline Circulatory & & & 0.69 & $0.82(0.80)$ \\
\hline Swelling in hands/feet & 0.90 & 0.09 & & \\
\hline Changes in smell/taste & 0.45 & 0.07 & & \\
\hline Hair thinning/loss & 0.72 & 0.12 & & \\
\hline Constipation & 0.62 & 0.10 & & \\
\hline Lymphedema & 0.52 & 0.09 & & \\
\hline Numbness hands/feet & 0.76 & 0.10 & & \\
\hline Sleep & & & 0.80 & $1.40(0.96)$ \\
\hline Waking too often & 1.34 & 0.09 & & \\
\hline Waking too early & 1.14 & 0.09 & & \\
\hline Difficulty falling asleep & 0.84 & 0.10 & & \\
\hline Vaginal discharge & 0.17 & 0.05 & & \\
\hline Hormonal & & & 0.88 & $0.89(0.76)$ \\
\hline Mood swings & 0.82 & 0.06 & & \\
\hline Depression & 0.97 & 0.07 & & \\
\hline Feeling anxious & 0.83 & 0.08 & & \\
\hline
\end{tabular}




\begin{tabular}{|c|c|c|c|c|}
\hline Factors/Items & Standardized Loading $a$ & Standard Error & Factor Alpha & Mean bother (SD) \\
\hline Nightmares & 0.47 & 0.07 & & \\
\hline Hot flashes & 0.50 & 0.11 & & \\
\hline Headaches & 0.50 & 0.08 & & \\
\hline Vaginal dryness & 0.35 & 0.10 & & \\
\hline Weight gain or loss & 0.71 & 0.11 & & \\
\hline
\end{tabular}

Note.

${ }^{a}$ All standardized loadings were significant at $\mathrm{p}<0.001$. 


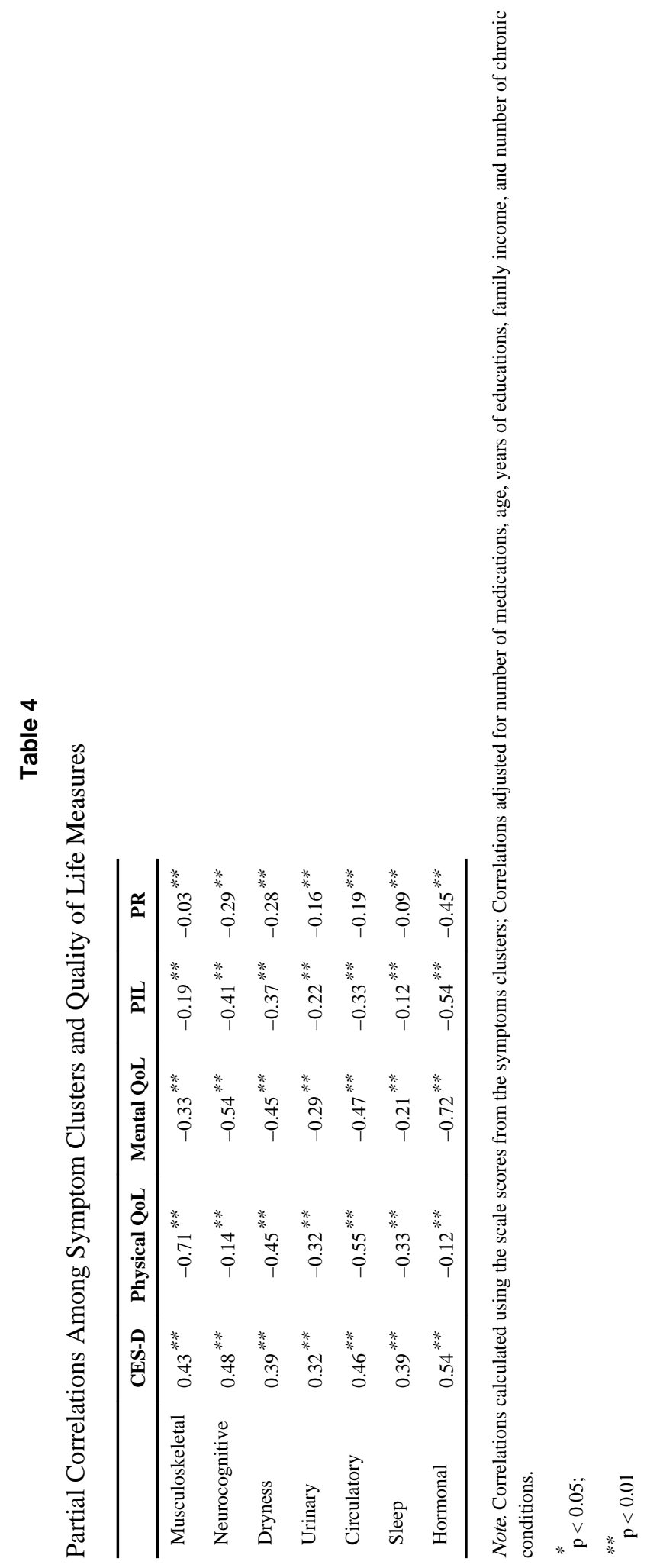

Oncol Nurs Forum. Author manuscript; available in PMC 2013 July 15. 